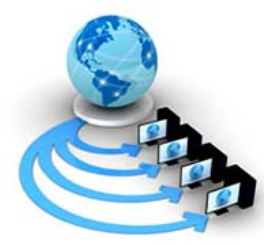

Volume 9, No. 3, May-June 2018

International Journal of Advanced Research in Computer Science

RESEARCH PAPER

Available Online at www.ijarcs.info

\title{
IoT ENABLED TRAFFIC CONTROL MODEL USING RASPBERRY PI
}

\author{
Syed Mohd Faisal Malik \\ School of Engineering and Science Technology \\ Jamia Hamdard \\ New Delhi, India
}

\author{
Md. Tabrez Nafis \\ School of Engineering and Science Technology \\ Jamia Hamdard \\ New Delhi, India
}

\author{
Mohd Hamzah Khan \\ School of Engineering and Science Technology \\ Jamia Hamdard \\ New Delhi, India
}

\begin{abstract}
In this paper a new methodology is described to efficiently handle and managed traffic in a highly populated and congested area. The traffic management system's framework make use of an essential technology required which is IoT .It also has other important parts such as an autonomous activity controller raspberry-pi, pi-camera, RFID ,IR sensors .To further help to navigate the traffic the proposed framework uses decisive algorithm and round-robin algorithm to find the Optimum path through traffic. Raspberry pi is used to manage all components collectively and individually. Total traffic in a region is determined by the help of IR sensors which uses RFID to distinguish high priority vehicles such as ambulance and VIPS from day to day traffic. There is an additional benefit of using RFID it can be used to locate the robbed or snatched vehicles. The proposed framework has two major parts i.e autonomous and manual. The algorithm used to navigate traffic play a vital part in making the proposed framework work efficiently.
\end{abstract}

Keywords: Raspberry pi , IR Sensor , RFID ,Optimizing algorithm , Manual Control

\section{INTRODUCTION}

IoT is a technology which is used by most of the metropolitan cities around the world to make their life more comfortable and easy, by using the technology of IoT , we can have the accurate result of what we wanted if we implemented the techniques correctly. With the help of IoT we can also store our personal or official database in our personal gadgets. Afterwards their stored database can be used for their further specifications. IOT eliminates the need to be close to operating a component, as components can be accessed and controlled through great distances. This makes the proposed framework more autonomous and reliable. Every distinct command can be used to command and control a particular area in IOT.

According to the Google report of most populated countries in the world India comes on $2^{\text {nd }}$ position after china with a total population of around 1,296,834,042 approx people and counting, so with this ranking in a populated country without any doubt there is an big issue of traffic gridlock on roads. There is a need of control framework for traffic control and movement in appropriate way such as congestion issues is eliminated. Subsequently by utilizing IOT framework idea this can be achieved. On the off chance that traffic lights work's contingent to the number of vehicles in a path or street, at that point the proper time control should be there for the traffic light so that the gridlock on roads should be reduced and every normal and crisis vehicles should pass without any problem Priority should be given to the crisis vehicle such as ambulance, fire brigade etc, next priority is given to the highly decorated government officials, and after that to the daily vehicles ,also if there is any possibility of robbed or snatched vehicles or disappeared vehicles, instead of complaining it to the nearest police station and waiting for the cops to take action ,we can use RFID(Radio frequency Identification) to take care of both the problems .

\section{LITERATURE SURVEY}

Traffic gridlock is a serious problem in metropolitan cities to combat this problem IoT combine with PIC microcontroller, IR sensors, optimizing algorithm and XBEE.IR sensors and XBEE need to perform cohesively so that the amount of traffic and light control can be easily managed by IR sensors and XBEE to identify high priority vehicles .Be that as it may, this plan was actualized as it were for programmed method of activity [1]. A calculation was additionally composed with the goal that maximum number of vehicles should pass in a given period of time. IR sensors read each RFID tag that passes through in front of it and categorizes it based on the priority system by which the vehicles are registered in traffic database such as high priority vehicles like ambulance, fire brigade, VIP's are given higher priority than day to day traffic [2] . In a traffic gridlock situation the IR sensors are important because it calculates the traffic density between the first sensor and last sensor and compares it with neighboring streets, if the traffic found in a street is greater than the prior threshold set than the green light is automatically turned on giving the gridlock area priority [4]. Shops and Malls also use RFID to keep track of their inventory so that no item can be taken out of store without billing .The same principle is applied for vehicles to track them in case of any matter and to also identify them as a priority vehicle or not [3] .Optimizing algorithm is also used to give the priority to the most congested direction so that the traffic will be controlled [5]. 


\section{PROPOSED METHODOLOGY}

In this proposed model we will give solution for gridlock Problem and also the identification and prioritization of Crisis vehicle can be done either autonomously or manually. The autonomous mode depends on output given by the IR sensor and applying optimizing algorithm such as decisive algorithm and round-robin algorithm to further simplify the process whereas in manual mode the traffic operator has the decision making ability by analyzing the traffic and managing it by himself. Raspberry pi is utilized as a part of framework it takes controls on all components of framework IR sensors are utilized to recognize the amount of traffic in a particular location. To give priority to emergency vehicle and for tracking lost cars RFID is utilized. Camera is utilized as a part of the manual mode of the proposed framework; it takes still pictures of traffic in real time. By observing these pictures authorized traffic management expert in control room will direct the traffic manually. In the next part we will have a look that how the color changing time algorithm according to the traffic, which will give the better result as compared to previous method, basically the algorithm is followed for only four directions, but in this we can change the number of directions according to the number of junctions present on road .

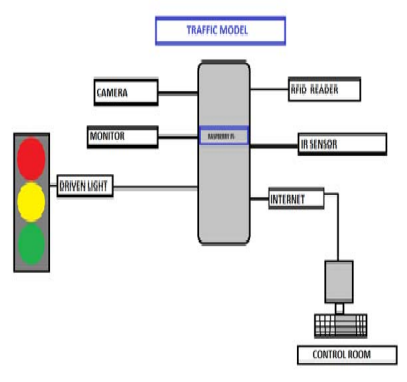

Fig- 1 Block Diagram

\section{A. Raspberry pi}

It is a debit card size board computer basically designs for the education purpose. When the raspberry pi was introduced there were no such functionalities of Bluetooth and Wi-Fi after seeing this as a drawback it was added afterwards. It also have video or audio jack which is very useful for analyzing the amount of gridlock present on the road. Raspberry pi manages each component separately as well as a whole. There are 3 generations of raspberry pi raspberry pi-1,2,3 , In our proposed system we have used raspberry pi -3 because raspberry pi-3 is $50 \%$ faster than other raspberry pi , current O.S is not compatible with raspberry pi 1 and 2 and raspberry pi 3 is also the latest one. It has Broadcom SOC and GPU. CPU'S speed should more than $600 \mathrm{M} \mathrm{Hz}$. RAM should be above $250 \mathrm{MB}$. It has SD card which have O.S there are six USB ports, CSI port is available to connect with camera. Raspberry-pi takes its power, input from USB cable as raspberry-pi also has audio and video jack . To connect to a bigger system .HDMI port is available and it also has GPIO.

\section{B. RFID}

In RFID technology information is interchange without any Physical connection of the components. RFID is combination of two parts RFID reader and RFID tag. RFID tags are of two types active and passive [8]. Active tags have continuous power supply where as passive tags will not have power supply, in the proposed framework passive tags are

Used. In passive tag when a tag is with into range then by mutual inductance the RFID tag will get recharge from the reader which has power supply. During mutual inductance the tag number present in the tag memory will be relate to the reader which will match it to the corresponding priority database of vehicles.

\section{IR Sensor}

Infra red rays cannot be seen by naked eye, IR sensor Makes use of infra-red rays .IR sensor is made of 2 parts Transmitter and Receiver [7]. The transmitter emits IR rays which are reflected back to the receiver if there is an object present in front of the IR sensor [10]. The distance of the object can also be calculated with respect to the IR sensor as Shown in the fig 2.

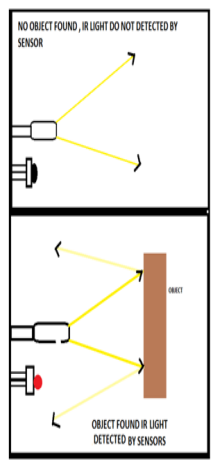

Fig. 2 shows how IR sensor works

\section{PI-Camera}

To take high quality pictures and video PI- camera is used at each traffic light; it uses a hard wire cable for connectivity to the CSI port of raspberry pi. Pi camera can also be used in road rage , theft to identify perpetrator in case of traffic accident. It is available near the traffic light as shown in fig 3 


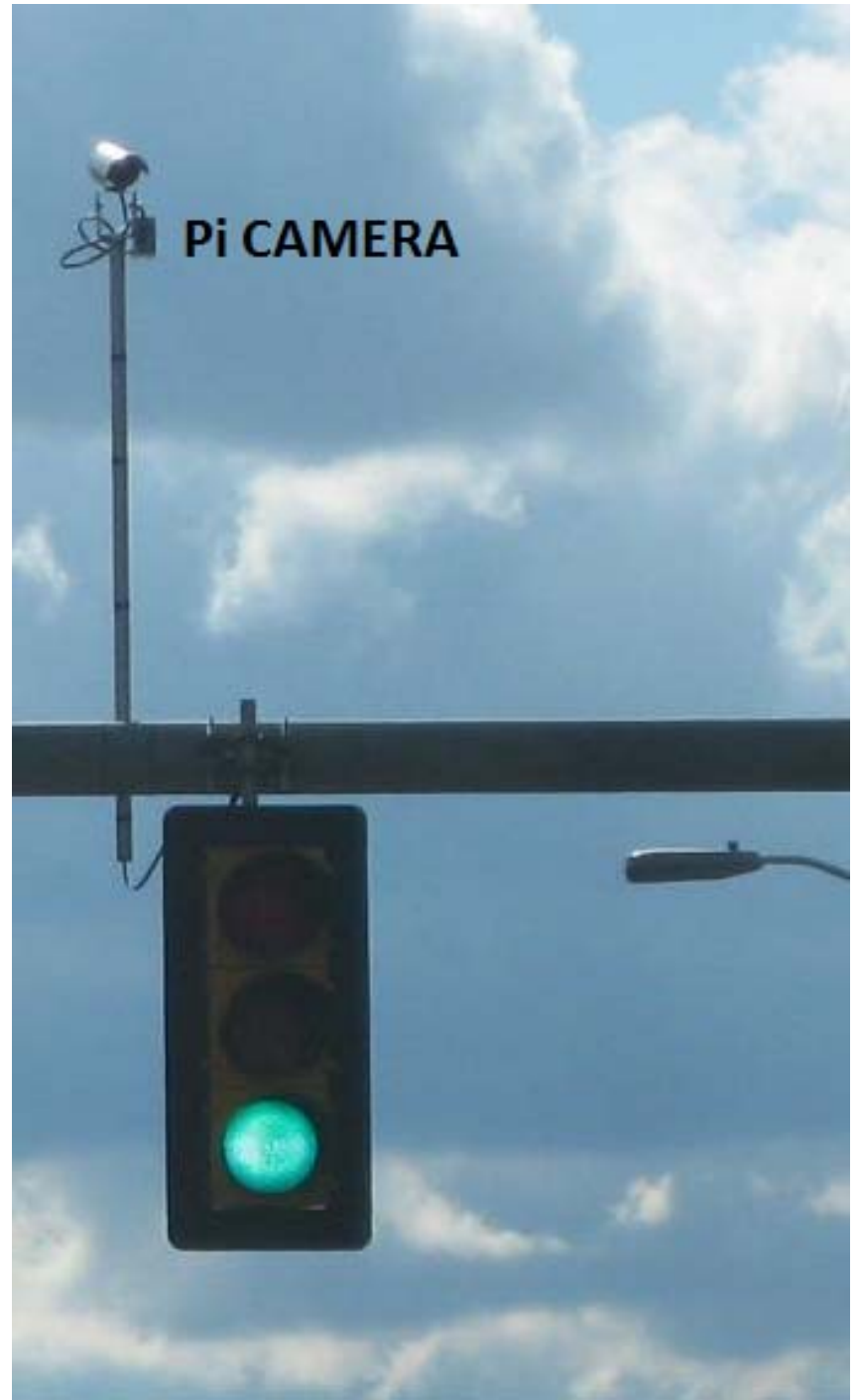

Fig 3 shows a PI -Camera attached with a Traffic light

\section{E. Driven light}

Driven lights are used as traffic signal to let the travellers know about the traffic movement. Driven lights are a diode which emits light in photon form as there are different colours present in the energy wavelength of the photon thus different colours of driven light can be used.

\section{F. Optimizing Algorithm}

The raspberry pi is having a coding function using python or embedded C programming languages as there is efficient programming language. With the help of using the optimizing algorithm which is decisive algorithm and round robin algorithm , the road which has more traffic will have a higher priority and a time delay is given to the vehicles to clear the road depending on the number of vehicles between first IR sensor and last IR sensor [9].

\section{LED OPTIMIZING ALGORITHM}

Step 1:- Set the Start time and End time of the traffic light counter for say from 5:00 am to 11:00 pm respectively

Step-2:- Initialize the LED counter value from 1 to 3 by increasing counter automatically.

Step -3:- Programs the counter to recognize the value-1 as RED signal

If counter $=1$

Calculate time delay for Green as number of vehicles between first IR sensor and last IR sensor multiplied by 5 seconds.

Green light active for time delay

Switch on yellow for 4 seconds and turn off green

signal

\section{Turn on Red Signal}

Step 4:- Calculate the above for Red light present at every direction North, south etc on the street

Step-5:- The algorithm will continuously check each direction LED lights to find a red light, if present it will repeat Step-3

Step-6:- This in turn would help manage traffic more effectively and the above algorithm optimizes it further.

This optimizing algorithm satisfies the proposed system. Optimizing algorithm which is Decisive Making algorithm

Causes the framework to take the decision relying on vehicles thickness whether to increase or reduce an opportunity to cross. Round robin algorithm is an optimizing algorithm which is used to handle the traffic LED lights and it is used to give Green signal to the road decided by the decisive algorithm and it will do it lastly until it satisfies the output.

\section{FEATURES}

The module comprises of one sensor area and raspberry pi organize which is utilized for observing and recognition reason to determine the amount of traffic in real time IR sensors are placed from the signal light to a certain " $x$ " distance behind the signal light to calculate the traffic density. The first IR sensor is kept perpendicular to the signal light " $x$ " meter, the second IR sensor is place at $(x+4)$ meter, and the third IR sensor at $(x+8)$ meter. If the output given by the IR sensor indicates high traffic density then the green light is turned on for a prior specified interval of time 


\section{CRISIS VEHICLE}

The traffic control room database contains all RFID card number present. As a high priority vehicle fitted with RFID tag passes through an IR sensor. The RFID tag number is compared against the RFID database to check whether it is a priority vehicle or not. If a priority vehicle is identified on a gridlock street than traffic lights will turn green for a pre specified amount of time. Every one of the area stretching over the span of the crisis vehicle taken the same strategy.

\section{BUS RAPID MANAGEMENT SYSTEM - FUTURE WORK}

The RFID reader is basically utilized for Metropolitan transport Corporation (MTC) digitalization. Each MTC transport will be labeled a different RFID label in view of the course they cover. The RFID reader will read and illuminate the closest transport station about the approaching transport. This information can be encouraged straightforwardly into cloud. A versatile application can be created which can be utilized by the client for better information on the running status of the transport thus benefiting in his/her time management.

\section{VEHICLE THEFT AND MANAGEMENT SYSTEM - FUTURE WORK}

Upon uncommon solicitations clients can likewise select their vehicle by the RFID labeling for singular consideration like vehicle observing and burglary avoidance [6]. The clients tag will be incorporated into the focal place for checking for exceptional practices or on the other hand for security. This gets an expansive database of vehicles in the city or entering or leaving the city. Crisis asks are additionally taken care of by the inside if a help flag is conveyed

\section{Manual Operation}

In manual operation the traffic control realize solely on the decision making ability of the traffic operator.The traffic operator will see the traffic through the Pi-camera and after carefully assessing the traffic situation . The traffic operator will take necessary steps to resolve the traffic gridlock issue manually

\section{CONCLUSION}

The proposed framework guarantees brilliant control Of movement framework and deals with the activity blockage. The outcome is as we anticipated. By this proposed framework time administration for flag lights is finished this implies discovery of ease of movement in volume at each intersection will decrease the activity gridlock issue. Freedom of activity for crisis car is effectively utilized. Subsequently, numerous valuable life would be spared. Furthermore, the framework has programmed and manual activity. All the framework control and discovery are conceivable by sensors and cameras set the intersections [10]. Also, at exhibit we have actualized the outline for just movement control, and vehicle leeway in the event of crisis. In future this can be stretched out to following of stolen car which can be simple and fast, when lost vehicle is distinguished an email might be dropped. In future it can also control the traffic through an optimizing algorithm in which it will work automatically by checking the density of the traffic in all directions and will act accordingly.

\section{REFERENCES}

[1] Bilal Ghazal, Khaled EIKhatib, Khaled Chahine, Mohamad Kherfan, " Smart traffic Light Control System ", IEEE 2016,PP 21-25

.[2] Abishek C, Mukul Kumar and Kumar Padmanabh "City Traffic Congestion Control in Indian Scenario using Wireless Sensors Network" ,PP 7-8, 2015

[3] Ayush Kr. Mittal and Deepika Bhandari, " A Novel Approach to Implement Green Wave system and Detection of Stolen Vehicles", 978-1-4673-4529- 3/12/\$31.00_c 2012 IEEE

[4] Rahul B. Pendor, P. P. Tasgaonkar " An IoT Framework for Intelligent vehicle monitoring System ", International Conference on Communication and Signal Processing, April 68, 2016, India

[5] K. Kishore Kumar, S. Durai, M. Thanjai Vadivel and K. Antony Kumar "Smart Traffic System using Raspberry Pi by Applying Dynamic Color Changer Algorithm”2-3,2016

.[6] ARanganath, T SreeValli "Intellgent MangementSystem For Density Based Control, Stolen Vehicle Auto Clerance, Aug 2015.,7,PP 12-15

[7] Faisal A. Al- Nasser,HosamRowaihy "Simulation of Dynamic Traffic control system based on Wireless sensor network", IEEE Symposium on Computers \& Informatics 2011, PP 40-45.

[8] Kumar S., Abhilash P.G., Jyothi D.G., Varaprasad G., "Violation Detection Method for Vehicular Ad Hoc Networking”, ACM/Wiley Security and Communication Networks, 2014, DOI:10.1002/sec.427.

[9] Varaprasad G., Wahidabanu R.S.D., "Flexible Routing Algorithm for Vehicular Area Networks”, in Proc. IEEE Conference on Intelligent Transport Systems Telecommunications, Osaka, Japan, PP.30-38, 2010.

[10] Xu Li, Wei Shu, Minglu Li, Hong-Yu Huang, Pei-En Luo, Min-You Wu, "Performance Evaluation of

Vehicle-Based Mobile Sensor Networks for Traffic Monitoring” IEEE transactions on vehicular technology, May 2009, vol. 58, no. 4, pp. 1647-1653 